\title{
SEARCHING FOR Be STARS IN THE OPEN CLUSTER NGC 663
}

\author{
P. C. Yu (俞伯傑 $)^{1}$, C. C. LIN (林建爭 $)^{1}$, W. P. CHEN (陳文屏 $)^{1,2}$, C. D. LEE (李建德 $)^{1}$, W. H. IP (葉永烜 $)^{1,3}$, \\ C. C. NGEOW (饒兆聰) ${ }^{1}$, Russ LAHER (良主嶺亜) ${ }^{4}$, JASON SuraCE ${ }^{4}$, AND SHRINIVAs R. KulKarNi ${ }^{5}$ \\ ${ }^{1}$ Graduate Institute of Astronomy, National Central University, 300 Jhongda Road, Jhongli 32001, Taiwan \\ ${ }^{2}$ Department of Physics, National Central University, 300 Jhongda Road, Jhongli 32001, Taiwan \\ ${ }^{3}$ Institute of Space Science, National Central University, 300 Jhongda Road, Jhongli 32001, Taiwan \\ ${ }^{4}$ Spitzer Science Center, California Institute of Technology, M/S 314-6, Pasadena, CA 91125, USA \\ ${ }^{5}$ Division of Physics, Mathematics and Astronomy, California Institute of Technology, Pasadena, CA 91125, USA \\ Received 2014 August 8; accepted 2014 October 14; published 2015 January 7
}

\begin{abstract}
We present Be star candidates in the open cluster NGC 663, identified by $\mathrm{H} \alpha$ imaging photometry with the Palomar Transient Factory Survey, as a pilot program to investigate how the Be star phenomena, the emission spectra, extended circumstellar envelopes, and fast rotation, correlate with massive stellar evolution. Stellar membership of the candidates was verified by 2 MASS magnitudes and colors and by proper motions (PMs). We discover four new Be stars and exclude one known Be star from being a member due to its inconsistent PMs. The fraction of Be stars to member stars $[\mathrm{N}(\mathrm{Be}) / \mathrm{N}$ (members)] in NGC 663 is $3.5 \%$. The spectral type of the $34 \mathrm{Be}$ stars in NGC 663 shows bimodal peaks at B0-B2 and B5-B7, which is consistent with the statistics in most star clusters. Additionally, we also discover 23 emission-line stars of different types, including non-member Be stars, dwarfs, and giants.
\end{abstract}

Key words: galaxies: star clusters: individual (NGC 663) - stars: emission-line, Be

\section{INTRODUCTION}

Emission-line stars are symbolized by Balmer lines, particularly the $\mathrm{H} \alpha$ lines in emission spectra. In general, the mechanisms responsible for the emission lines include mass accretion and chromospheric activity. Emission stars can be in almost any stage during stellar evolution, from pre-mainsequence T Tauri and Herbig Ae/Be stars, main-sequence Be/ $\mathrm{Ae}$ and $\mathrm{dMe}$ stars, and Wolf-Rayet stars to giants and supergiants. Among these, the classical Be stars are particularly interesting because, other than the emission spectra, they also show near-infrared-excess emission above the stellar photospheric radiation, and fast rotation with an equatorial speed up to $70 \%-80 \%$ of breakup velocity (Townsend et al. 2004).

The infrared excess of Be stars is usually attributed to plasma free-free emission in the extended envelopes. Even though it is thought that the fast rotation must play a decisive role, the interplay of the Be phenomena, namely the emission lines, infrared excess, and rapid rotation, is still unclear. One school of thought proposes that classical Be stars become fast rotators only at the second half of the main sequence (Fabregat \& Torrejón 2000), or near the turn-off (Keller et al. 2001). These Be stars are found in young $(\sim 10 \mathrm{Myr})$ star clusters in the Milky Way galaxy (Mathew et al. 2008), LMC, and SMC (Wisniewski \& Bjorkman 2006). Alternatively, the stars might have been spun up by mass transfer in binaries (McSwain \& Gies 2005).

Care should be exercised when collecting the sample of classical Be stars in a young cluster because a distinctly different group, the Herbig Ae/Be stars, intermediate-mass premain sequence objects, exhibit very similar observational properties, such as their emission spectra and infrared excess. Lee \& Chen (2011) analyzed a group of Be stars away from any signposts of recent star formation, thereby being excluded from the pre-main sequence status, yet have unusually large infrared excess, with observed 2MASS colors, $J-H$ and
$H-K_{s}$, both greater than $0.6 \mathrm{mag}$, which must be accounted for by thermal dust emission.

The primary uncertainty in the age determination of a single Be star is rectified if Be stars in a sample of star clusters at different ages can be studied. However, the sample of classical Be stars in open clusters is not complete due to several reasons. First, a nearby open cluster occupies a wide sky area, so a comprehensive survey for emission-line stars is time consuming, and often limited to bright stars. Secondly, Be stars show photometric and spectroscopic variability. With the wide field coverage of the Palomar Transient Factory (PTF) project, we have initiated a program to search for emission stars in clusters of different ages. This paper reports our methodology to identify emission-line stars with the PTF $\mathrm{H} \alpha$ images.

Here we present the results for the first target, the young open cluster NGC $663\left(\alpha_{2000}=01^{\mathrm{h}} 46^{\mathrm{m}} 12^{\mathrm{s}}, \delta_{2000}=61^{\circ} 13^{\prime} 30^{\prime \prime}\right.$; galactic coordinates $\left.l, b=129.470^{\circ},-0.953^{\circ}\right)$. With an age of 31 Mys and at a distance of $2.1 \mathrm{kpc}$ (Kharchenko et al. 2013), this cluster is known to host a large number of Be stars (Sanduleak 1979; Pigulski et al. 2001; Mathew \& Subramaniam 2011). NGC 663 was also considered to be a part of the stellar association Cas OB8. Cas OB8 has been reported to be located at a distance of $2.9 \mathrm{kpc}$ (Dambis et al. 2001), and thus NGC 663 appears to be in the foreground. However, the distance of Cas OB8 seems to be overestimated. Using old and new reduction of the Hipparcos data, the mean distance of Cas OB8 is suggested to be $2.3 \mathrm{kpc}$ (Mel'nik and Dambis 2009). Taking the possible uncertainty of the distance estimation into consideration, NGC 663 could be a part of Cas OB8.

In Section 2, we describe the acquisition of the observations and the analysis to recognize emission stars. In Section 3, we present our list of $\mathrm{H} \alpha$ stars and compare our results, which reach fainter magnitudes, with those in the literature. Section 4 gives a summary of this study. 


\section{OBSERVATIONS AND DATA ANALYSIS}

Data used in this study include the PTF $\mathrm{H} \alpha$ images used to identify the emission star candidates and the 2MASS nearinfrared photometry and proper motions (PMs) used to characterize the emission star candidates. The PTF is an automated, wide-field survey with a 7.3 square degree field of view (Law et al. 2009). All observations were carried out with the 48 inch Samuel Oschin Telescope at Palomar Observatory. The $\mathrm{H} \alpha$ and the continuum images are taken through HA 656 and HA 663 narrow-band filters (hereafter the $\mathrm{H} \alpha$ and $\mathrm{r}$ band, respectively), which have a central wavelength of $6563 \AA$ and $6630 \AA$, with a bandwidth of $75 \AA$. The exposure time for the $\mathrm{H} \alpha$ - and r-band images is $60 \mathrm{~s}$. The image data were processed for bias corrections, flat fielding, and astrometric calibration with pipelines developed by the Infrared Processing and Analysis Center (IPAC; Laher et al. 2014).

The 2MASS point source catalog (Cutri et al. 2003) provides a uniformly calibrated database of the entire sky in the near-infrared $J, H$, and $K_{s}$ bands with a $10 \sigma$ detection limit of $15.8,15.1$, and $14.3 \mathrm{mag}$, respectively. Kinematic information can be obtained to secure the membership in a star cluster by the PPMXL data set, which is derived from an all-sky merged catalog based on the USNO-B1 and 2MASS positions of 900 million stars and galaxies, reaching a limiting $\mathrm{V} \sim 20 \mathrm{mag}$ (Röser et al. 2010). The typical error of PMs is less than 2 mas per year for the brightest stars with Tycho-2 $\left(\mathrm{H} ø \mathrm{~g}\right.$ et al. 2000) observations and is more than 10 mas year $^{-1}$ at the faint limit. However, Röser et al. (2010) noted that about 90 million (10\%) objects of PPMXL include spurious entries; they found double or several matches of USNO-B1 stars with a 2MASS star. Kharchenko et al. (2012) have averaged such PMs and computed their errors in sky areas with star clusters including NGC 663. Therefore, we use these PMs instead of PPMXL data.

In our study, we made use of the 2MASS photometry and the PMs to select and characterize stellar member candidates. While matching counterparts in different star catalogs, a coincidence radius of $2^{\prime \prime}$ was used among PTF, 2MASS, and PMs sources.

\subsection{Data Analysis}

\subsubsection{The Search Region}

Open clusters generally have irregular shapes during their evolution through either the internal relaxation process or Galactic external perturbation (Chen et al. 2004). We therefore need to adopt a suitable region to include all possible member stars for NGC 663. To determine the survey region, we selected the 2MASS point sources with $\mathrm{S} / \mathrm{N} \geqslant 10$ in all $J, H, K_{S}$ bands and with PM uncertainties $<5.0$ mas year $^{-1}$. Based on the radial density profile (Figure 1), the best half-Gaussian fitting gives a $3 \sigma$ diameter of $\sim 30^{\prime}$, which corresponds to a diameter of $\sim 18 \mathrm{pc}$ at $2.1 \mathrm{kpc}$. Hence we adopted a box with a side of $40^{\prime}$ $(4 \sigma)$ as the field for NGC 663 in the subsequent analysis to cover the region of previous studies and possible candidates.

\subsubsection{Ho Photometry and Emission-line Candidates}

To identify possible $\mathrm{H} \alpha$ emission stars, we compute the difference in the instrumental magnitude between the $\mathrm{H} \alpha$ - and the r-band images (e.g., $\mathrm{r}-\mathrm{H} \alpha$ ). Under the assumption that the majority of stars in the field exhibit $\mathrm{H} \alpha$ neither in emission nor in absorption, those that stand out in $\mathrm{r}-\mathrm{H} \alpha$ are probable $\mathrm{H} \alpha$ stars. Figure 2 shows the $\mathrm{r}-\mathrm{H} \alpha$ values for all stars (gray dots) within a field of $\sim 2^{\circ} \times 1^{\circ}$, which guarantees coverage of the spatial extent of NGC 663. Because of the increased scattering of the $\mathrm{r}-\mathrm{H} \alpha$ values toward faint magnitudes due to photometric errors, we exercised different selection criteria according to the brightness of the stars. We first calculated the photometric scattering $\left(\sigma_{p}\right)$ for each $0.5 \mathrm{mag}$ r-band bin with the error propagated from photometric and systematic errors. The photometric error is the weighted photometric error of $\mathrm{r}-\mathrm{H} \alpha$ values for stars within the $0.5 \mathrm{mag}$ bin while the systematic errors are the standard deviation of $\mathrm{r}-\mathrm{H} \alpha$ values for stars within the same bin. Then we selected emission-line candidates for those with $\mathrm{r}-\mathrm{H} \alpha>2 \sigma_{p}$ in each $0.5 \mathrm{mag}$ bin, i.e., with significant flux excess in the $\mathrm{H} \alpha$ image than in the r-band image, as illustrated in Figure 2. A total of 42 emission-line candidates were thus identified within a $40^{\prime} \times 40^{\prime}$ field. Table 1 lists the properties of the 42 emission-line candidates. The first column provides identification numbers. Columns 2 and 3 provide coordinates in degrees. Columns 4 and 5 show the PM measurements in R.A. and decl. Column 6 gives PM uncertainties. Subsequent columns, 7-9, list the 2MASS $K_{s}$, $J-H$, and $H-K_{s}$ values. Column 10 lists names of known Be stars. Columns 11 and 12 give the PTF instrumental r-band and $\mathrm{r}-\mathrm{H} \alpha$ magnitudes. Column 13 provides the object classifications adopted from SIMBAD. Figure 3 shows the spatial distribution of our candidates.

\subsubsection{Identification of Membership}

We verify member stars of NGC 663 with photometric and kinematic memberships. First, we determine the photometric membership by selecting stars within the region that are near the isochrone (Girardi et al. 2002) in the $K_{s}$ versus $J-K_{s}$ color-magnitude diagram (CMD). Pandey et al. (2005) suggest a range of age from 10 to $50 \mathrm{Myr}$ for NGC 663. Here we adopt the mean age of $\sim 31 \mathrm{Myr}$, the distance of $2.1 \mathrm{kpc}$, and $\mathrm{E}(\mathrm{B}-\mathrm{V})$ of $0.7 \mathrm{mag}$ from Kharchenko et al. (2013) for the isochrone. The region for the photometric membership is defined by estimating $J-K_{s}$ errors propagated from photometric errors of $J$ and $K_{s}$ values along with the isochrone from $K_{s}=7 \mathrm{mag}$ toward faint $K_{s}$ magnitudes (Figure 4). On the other hand, verification of the photometric membership for the $\mathrm{H} \alpha$ emission-line candidates is not intuitive. Lee \& Chen (2011) showed that Be stars can have unusually large infrared excess with $J-H$ and $H-K_{s}$ both greater than $0.6 \mathrm{mag}$. As shown in Figure 4, we thus extend the selection region of the photometric membership with $J-K_{s} \sim 1.2 \mathrm{mag}$ for the emission-line candidates.

Secondly, we define a region that could include the possible kinematic membership. We have calculated the averaged PM and standard deviations of stars within the $40^{\prime} \times 40^{\prime}$ field by fitting a Gaussian distribution to PMs. The mean PM is $(-0.80$, -2.43) mas year ${ }^{-1}$, and standard deviations $\sigma_{\mu \alpha}$ and $\sigma_{\mu \delta}$ are 3.58 mas year $^{-1}$ and 3.41 mas year $^{-1}$, respectively. We then adopt a $2 \sigma$ approach to determine the kinematic membership. The sigma $\sigma_{\mu}=4.94$ mas year $^{-1}$ is the error propagated from $\sigma_{\mu \alpha}$ and $\sigma_{\mu \delta}$. Stars are considered to have kinematic membership if they are located inside the $2 \sigma_{\mu}$ region (e.g., $2 \times \sigma_{\mu}$ $=9.88$ mas year $^{-1}$; Figure 5).

Finally, objects are considered to be member stars according to their photometric membership as well as their kinematic membership. Based on the CMD and PM analysis, we identify 


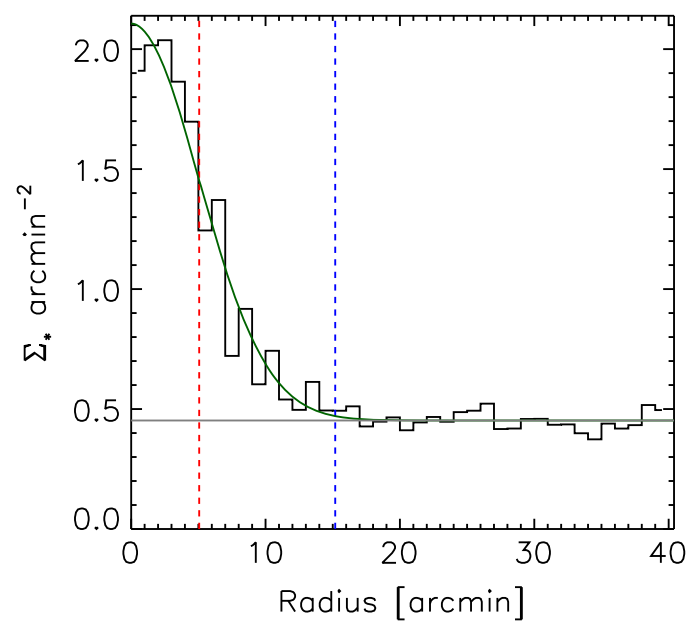

Figure 1. Radial density profile for NGC 663. The gray horizontal line depicts the background density. The green curve shows the best Gaussian fitting results. Red and blue vertical lines represent the width of the curve with $1 \sigma$ and $3 \sigma$, respectively. We adopted a box with a side of $40^{\prime}(4 \sigma)$ for the field of NGC 663 to cover the region of previous studies and possible candidates.

959 member stars in total within the $40^{\prime} \times 40^{\prime}$ field. Among the 42 emission-line candidates, 23 objects meet our criteria and are suggested to be member stars.

To further select possible Be stars from these 23 emissionline candidates, we defined a region in the $J-H$ versus $H-K_{s}$ color-color diagram, which could cover $99 \%$ of $\mathrm{Be}$ stars collected from the literature (Zhang et al. 2005). As shown in Figure 6, gray contours represent over 1000 Be stars. Assuming that most Be stars have similar infrared colors, we thus selected possible Be stars inside the gray-dashed region in the color-color diagram. Finally, 19 candidates are selected as Be stars in NGC 663. Among the 19 candidates, we have confirmed that 15 objects are previously reported Be stars. Therefore, we discover four new Be stars (ID number: 12, 35, 39, and 41) in NGC 663.

\section{RESULTS AND DISCUSSION}

\subsection{Known Be Stars}

Pigulski et al. (2001) listed the compilation of 25 known Be stars in NGC 663 from previous studies. They further discovered four new Be stars using $\mathrm{H} \alpha$ photometry with a $60 \mathrm{~cm}$ telescope and presented a list of 29 Be stars in the $30^{\prime} \times 30^{\prime}$ field. Mathew \& Subramaniam (2011) found 2 more new Be stars with spectroscopic observations and increased the number to 31 . We list the properties of these 31 known Be stars in Table 2. The first column provides the names of known Be stars. Columns 2 and 3 provide coordinates in degrees. Columns 4 and 5 list the PM measurements in R.A. and decl. adopted from Kharchenko et al. (2012). Column 6 show errors in the PM measurements. Subsequent columns, 7, 8, and 9, list the 2MASS $K_{s}, J-H$, and $H-K_{s}$ values. Column 10 shows the $\mathrm{H} \alpha$ equivalent widths $(\mathrm{EW}(\mathrm{H} \alpha))$ adopted from Mathew \& Subramaniam (2011). We use the averaged $\mathrm{EW}(\mathrm{H} \alpha)$ if the $\mathrm{Be}$ stars had multiple observations. Column 11 indicates the spectral type adopted from Mathew \& Subramaniam (2011). The final column shows the notes of the stars.

Among 31 known Be stars, we re-identify 15 objects and misidentify 6 objects. The other 10 objects are discounted because of photometric problems, including saturation,

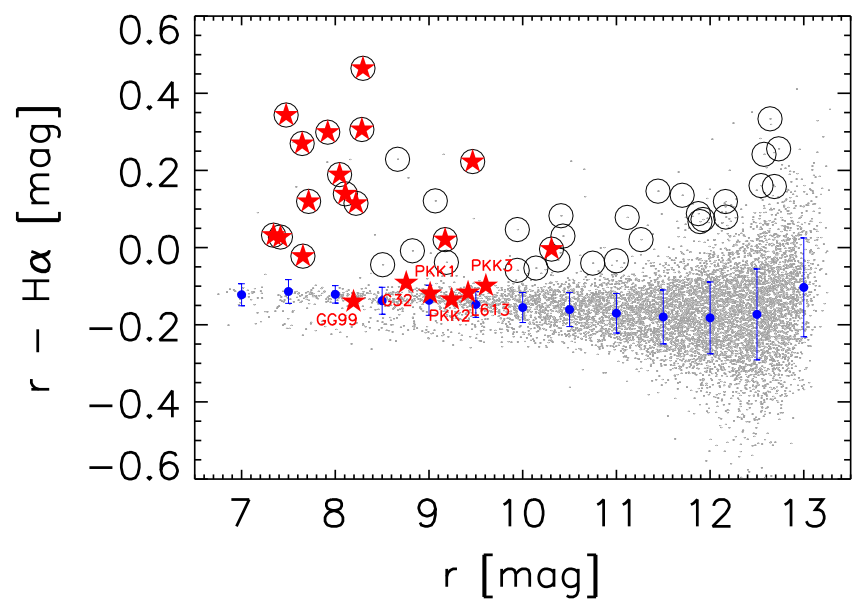

Figure 2. $\mathrm{H} \alpha$ photometry. Gray dots: all stars within a field of $2^{\circ} \times 1^{\circ}$; open circles: emission-line candidates within a field of $40^{\prime} \times 40^{\prime}$; red pentagrams: known Be stars in NGC 663. Blue filled circles represent mean values of $\mathrm{r}-\mathrm{H} \alpha$ values within each $0.5 \mathrm{mag}$ r-band bin, and the photometric scattering are calculated with the error propagated from photometric and systematic errors The photometric error is the weighted photometric error of $\mathrm{r}-\mathrm{H} \alpha$ values for stars within the $0.5 \mathrm{mag}$ bin while the systematic errors are the standard deviation of $\mathrm{r}-\mathrm{H} \alpha$ values for stars within the same bin. PKK 1, PKK 2, PKK 3, GG 99, G32, and L613 are known Be stars that are not selected by our method.

blending of nearby stars, and contamination of the CCD gap. The misidentification of six objects might be caused by variability; we find that two of the misidentified objects, GG 99 and L613, are suggested to show $\mathrm{H} \alpha$ variability (Sanduleak 1990; Mathew \& Subramaniam 2011). Another reason for the misidentification might be due to weak emissions. As shown in Figure 2, the four misidentified objects (G32, PKK 1, PKK 2, and PKK 3) show low $\mathrm{r}-\mathrm{H} \alpha$ values, which are similar to the mean values. This result indicates that their $\mathrm{H} \alpha$ strength could be too weak for firm identification. Figure 7 shows the correlation between the $\mathrm{EW}(\mathrm{H} \alpha)$ and $\mathrm{r}-\mathrm{H} \alpha$ values, suggesting the $\mathrm{EW}(\mathrm{H} \alpha)$ of the four misidentified objects are weaker than $-10 \AA$. Therefore, the misidentification of the four objects could be due to the weak emissions. If we select emission-line candidates with a lower threshold $\mathrm{r}-\mathrm{H} \alpha>1 \sigma_{p}$ to include objects with weak $\mathrm{H} \alpha$, we then have an additional 124 emission-line candidates with lower confidence levels while only 2 more known Be stars (G32 and PKK 3) can be identified as emission-line stars. Thus, our detection limit for emissionline candidates is about $\mathrm{EW}(\mathrm{H} \alpha)=-10 \AA\left(\mathrm{r}-\mathrm{H} \alpha>2 \sigma_{p}\right)$.

\subsection{New Be Candidates}

We discover four possible new Be stars (ID number: 12, 35, 39 , and 41) with $\mathrm{r}-\mathrm{H} \alpha>0.12$. The $\mathrm{EW}(\mathrm{H} \alpha)$ versus $\mathrm{r}-\mathrm{H} \alpha$ correlation (Figure 7) shows these newly discovered Be stars to have $\mathrm{EW}(\mathrm{H} \alpha)$ stronger than $-30 \AA$, thus the undetection of the four new Be stars in previous studies should not be caused by weak emissions. In addition to the emission-line variability, the non-detection of the four new Be stars in previous studies might be due to their positions. Previous surveys of NGC 663 covered less than the central $30^{\prime} \times 30^{\prime}$ field, whereas two of the four new Be stars are located outside this region. Besides, by the same diagnosis, we find that one previously claimed Be star, SAN 28, should not be a member star due to its inconsistent $\mathrm{PM}\left(\mu_{\alpha}=-16.07 \pm 3.95, \mu_{\delta}=-34.09 \pm 3.95\right.$ mas year $\left.{ }^{-1}\right)$. We thus conclude that the number of Be stars in NGC 663 is 34 . 
Table 1

Emission-line Candidates

\begin{tabular}{|c|c|c|c|c|c|c|c|c|c|c|c|c|}
\hline ID & $\begin{array}{l}\text { R.A. } \\
\text { (deg) }\end{array}$ & $\begin{array}{l}\text { Decl. } \\
\text { (deg) }\end{array}$ & $\begin{array}{c}\mu_{\alpha} \\
\left(\text { mas year }^{-1}\right)\end{array}$ & $\begin{array}{c}\mu_{\delta} \\
\left(\operatorname{mas} \operatorname{year}^{-1}\right)\end{array}$ & $\begin{array}{c}\text { epm } \\
\left(\text { mas year }^{-1}\right)\end{array}$ & $\begin{array}{c}K_{s} \\
(\mathrm{mag})\end{array}$ & $\begin{array}{c}J-H \\
(\mathrm{mag})\end{array}$ & $\begin{array}{c}H-K_{s} \\
(\mathrm{mag})\end{array}$ & Name & $\begin{array}{c}\mathrm{r} \\
(\mathrm{mag})\end{array}$ & $\begin{array}{c}\mathrm{r}-H \alpha \\
(\mathrm{mag})\end{array}$ & SIMBAD \\
\hline 1 & 26.483740 & 61.212574 & -1.35 & 4.68 & 1.50 & 9.675 & 0.266 & 0.321 & GC 97 & 7.715 & 0.120 & $\mathrm{Be}$ \\
\hline 2 & 26.443295 & 61.155804 & -1.79 & -1.36 & 2.00 & 9.895 & 0.198 & 0.228 & GG 95 & 7.647 & 0.270 & $\mathrm{Be}$ \\
\hline 3 & 26.326494 & 60.969196 & -4.48 & 2.90 & 2.00 & 9.751 & 0.226 & 0.278 & GG 90 & 7.415 & 0.027 & $\mathrm{Be}$ \\
\hline 4 & 26.750830 & 61.356567 & -2.56 & -1.37 & 2.00 & 9.486 & 0.200 & 0.213 & GG 104 & 7.341 & 0.032 & HXB \\
\hline 5 & 26.861519 & 61.145599 & -3.74 & -6.38 & 2.00 & 10.237 & 0.158 & 0.033 & GG 108 & 7.476 & 0.344 & $\mathrm{Be}$ \\
\hline 6 & 26.739870 & 61.027885 & -5.86 & 0.82 & 2.00 & 9.937 & 0.189 & 0.233 & GG 103 & 7.655 & -0.022 & $\mathrm{Be}$ \\
\hline 7 & 26.415096 & 61.216434 & -2.40 & 3.04 & 2.00 & 10.494 & 0.191 & 0.238 & GG 94 & 8.107 & 0.139 & $\mathrm{Be}$ \\
\hline 8 & 26.407560 & 61.133095 & 0.63 & -2.93 & 2.00 & 10.644 & 0.167 & 0.291 & GG 93 & 7.920 & 0.299 & $\mathrm{Be}$ \\
\hline 9 & 26.765589 & 61.292236 & -3.92 & 0.93 & 2.00 & 10.534 & 0.234 & 0.207 & D01-034 & 8.221 & 0.115 & $\mathrm{Be}$ \\
\hline 10 & 26.627619 & 61.241455 & -7.84 & -1.38 & 2.00 & 10.463 & 0.165 & 0.196 & VES 620 & 8.046 & 0.189 & $\mathrm{Be}$ \\
\hline 11 & 26.202705 & 61.215088 & 1.04 & -2.00 & 5.00 & 7.284 & 1.104 & 0.367 & $\ldots$ & 8.505 & -0.044 & $\ldots$ \\
\hline 12 & 26.108356 & 60.895775 & 2.89 & 1.26 & 2.00 & 11.642 & 0.090 & 0.073 & $\ldots$ & 8.665 & 0.229 & $\ldots$ \\
\hline 13 & 26.914015 & 61.305721 & -3.06 & -0.88 & 2.79 & 10.144 & 0.292 & 0.298 & GG 109 & 8.295 & 0.465 & $\mathrm{Be}$ \\
\hline 14 & 26.648338 & 61.227543 & -4.11 & 2.39 & 2.00 & 10.126 & 0.318 & 0.318 & GG 101 & 8.284 & 0.306 & $\mathrm{Be}$ \\
\hline $15^{*}$ & 26.320665 & 61.123646 & -98.90 & -8.50 & 12.90 & 9.852 & 0.716 & 0.158 & $\ldots$ & 8.823 & -0.008 & iC \\
\hline 16 & 26.068714 & 61.076244 & -0.98 & 2.74 & 5.00 & 7.892 & 1.115 & 0.345 & $\ldots$ & 9.185 & -0.038 & $\ldots$ \\
\hline $17 *$ & 26.444133 & 60.972193 & 357.10 & 53.10 & 5.00 & 9.565 & 0.571 & 0.240 & $\ldots$ & 9.068 & 0.121 & $\mathrm{PM}$ \\
\hline 18 & 26.558376 & 61.228851 & -8.65 & 0.34 & 2.00 & 11.168 & 0.177 & 0.288 & BG 114 & 9.172 & 0.021 & $\mathrm{Be}$ \\
\hline 19 & 26.748318 & 61.208199 & -5.21 & 2.70 & 2.59 & 11.590 & 0.227 & 0.215 & VES 624 & 9.466 & 0.223 & $\mathrm{Be}$ \\
\hline 20 & 26.431408 & 61.415092 & -3.61 & -3.60 & 5.00 & 9.163 & 0.965 & 0.474 & $\ldots$ & 9.943 & 0.047 & $\ldots$ \\
\hline 21 & 26.230267 & 61.005119 & 9.26 & -11.48 & 3.90 & 11.725 & 0.585 & 0.106 & $\ldots$ & 9.941 & -0.059 & $\ldots$ \\
\hline 22 & 26.943848 & 61.370613 & 29.70 & -52.68 & 3.90 & 11.112 & 0.731 & 0.139 & $\ldots$ & 10.141 & -0.053 & $\ldots$ \\
\hline 23 & 26.036928 & 61.300438 & -1.89 & -0.89 & 5.00 & 7.337 & 1.400 & 0.549 & $\ldots$ & 10.409 & 0.083 & $\ldots$ \\
\hline 24 & 26.223892 & 61.233059 & -7.30 & -15.64 & 3.90 & 11.527 & 0.701 & 0.167 & $\ldots$ & 10.376 & -0.032 & $\mathrm{iC}$ \\
\hline 25 & 27.009970 & 61.320004 & 28.46 & -15.60 & 3.90 & 11.623 & 0.632 & 0.215 & $\ldots$ & 10.747 & -0.040 & $\ldots$ \\
\hline 26 & 26.823006 & 61.221569 & -2.82 & 0.51 & 3.90 & 12.619 & 0.194 & 0.148 & PKK 4 & 10.308 & -0.004 & $\mathrm{Be}$ \\
\hline 27 & 27.185598 & 60.894218 & 0.07 & -2.06 & 5.00 & 7.355 & 1.280 & 0.462 & $\ldots$ & 10.430 & 0.030 & $\ldots$ \\
\hline 28 & 26.424812 & 61.442692 & -3.41 & -1.26 & 3.90 & 12.887 & 0.511 & 0.133 & $\ldots$ & 11.000 & -0.034 & $\ldots$ \\
\hline 29 & 27.068419 & 61.100658 & -2.23 & -4.46 & 5.00 & 8.112 & 1.252 & 0.497 & $\ldots$ & 11.115 & 0.078 & $\ldots$ \\
\hline 30 & 26.023653 & 61.306664 & 24.85 & -19.31 & 4.00 & 11.954 & 0.691 & 0.195 & $\ldots$ & 11.444 & 0.146 & $\ldots$ \\
\hline 31 & 26.088741 & 61.198486 & 1.61 & 3.07 & 4.00 & 12.998 & 0.618 & 0.170 & $\ldots$ & 11.702 & 0.136 & $\ldots$ \\
\hline $32 *$ & 26.698837 & 61.124264 & -233.60 & -5.40 & 15.30 & 11.920 & 0.594 & 0.223 & $\ldots$ & 11.263 & 0.021 & $\mathrm{iC}$ \\
\hline 33 & 26.466228 & 61.297523 & -12.96 & -15.77 & 3.90 & 13.717 & 0.418 & 0.196 & $\ldots$ & 12.167 & 0.079 & $\mathrm{iC}$ \\
\hline 34 & 26.037413 & 61.119137 & 52.73 & -19.68 & 12.07 & 13.852 & 0.306 & 0.180 & $\ldots$ & 11.892 & 0.067 & $\ldots$ \\
\hline $35^{*}$ & 26.322468 & 61.047340 & 7.40 & 7.20 & 4.00 & 14.071 & -0.042 & 0.386 & $\ldots$ & 12.163 & 0.119 & $\ldots$ \\
\hline 36 & 26.706167 & 61.425907 & 9.88 & -23.59 & 3.90 & 12.303 & 0.623 & 0.242 & $\ldots$ & 11.875 & 0.088 & $\ldots$ \\
\hline 37 & 26.877998 & 61.107220 & -21.54 & -10.89 & 4.65 & 12.812 & 0.729 & 0.191 & $\ldots$ & 11.924 & 0.073 & $\ldots$ \\
\hline 38 & 26.213600 & 61.322948 & 26.75 & -37.80 & 15.86 & 13.727 & 0.613 & 0.182 & $\ldots$ & 12.575 & 0.242 & $\ldots$ \\
\hline 39 & 26.347054 & 61.265411 & 4.05 & 7.71 & 4.00 & 14.227 & 0.453 & 0.184 & $\ldots$ & 12.732 & 0.256 & $\ldots$ \\
\hline 40 & 26.411844 & 60.933365 & 45.93 & -61.68 & 3.90 & 13.666 & 0.703 & 0.113 & $\ldots$ & 12.640 & 0.334 & $\ldots$ \\
\hline 41 & 26.214262 & 60.938358 & -14.28 & -6.80 & 4.00 & 14.206 & 0.457 & 0.255 & $\ldots$ & 12.544 & 0.161 & $\ldots$ \\
\hline 42 & 26.976549 & 60.914070 & 62.77 & -33.19 & 5.79 & 13.935 & 0.528 & 0.118 & $\ldots$ & 12.686 & 0.158 & $\ldots$ \\
\hline
\end{tabular}

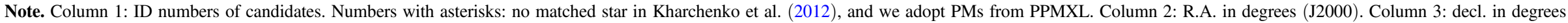

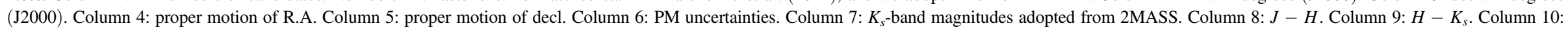

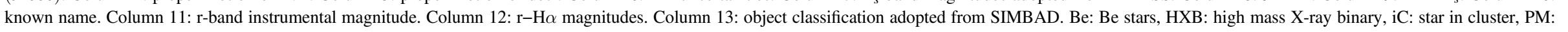
high proper-motion star. 


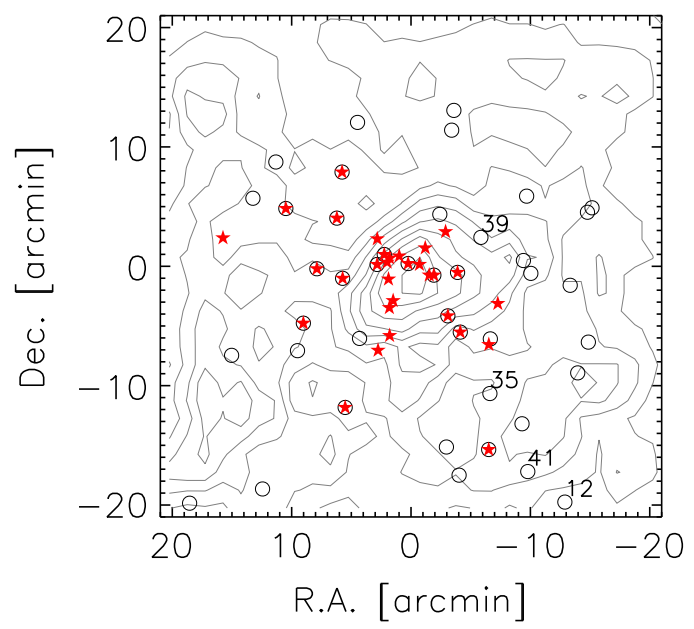

Figure 3. Spatial distribution of known Be stars and emission-line candidates. Symbols are the same as in Figure 2. The symbols with numbers represent ID numbers of four newly discovered Be stars. Contours represent the distribution of member stars within a field of $40^{\prime} \times 40^{\prime}$.

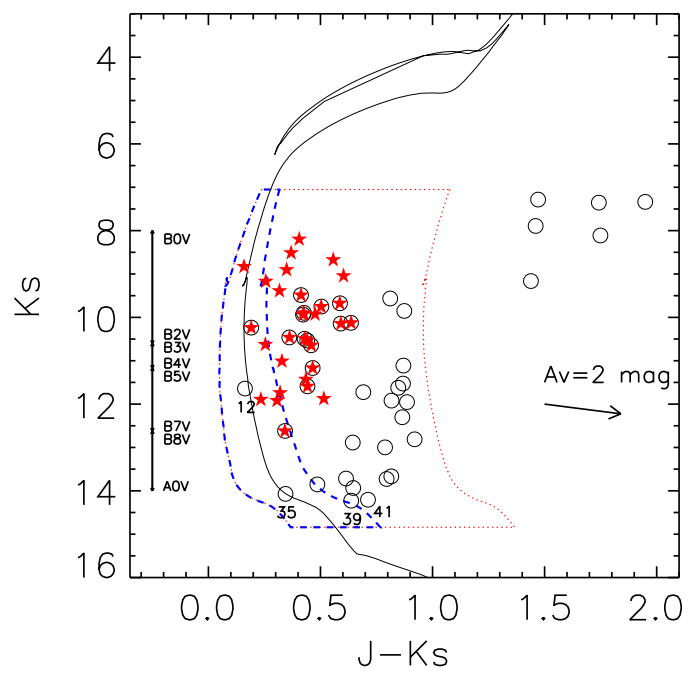

Figure 4. 2MASS color-magnitude diagram in the cluster region. The black solid line delineates the post-main-sequence isochrone (Girardi et al. 2002) for $31 \mathrm{Myr}$ located at $2.1 \mathrm{kpc}$ (Kharchenko et al. 2013) with an interstellar extinction of $A_{V} \sim 2.1 \mathrm{mag}$. Symbols are the same as in Figure 2. Nonemission-line stars within the blue-dashed region are considered to have photometric membership. Emission-line candidates are considered to have photometric membership within the wider red-dashed region.

\subsection{Be Star Fraction}

Mathew et al. (2008) discovered 22 Be candidates among a total of 486 B-type stars in NGC 663. They further compared the ratio of $\mathrm{Be}$ stars to $\mathrm{B}$-type stars $[\mathrm{N}(\mathrm{Be}) / \mathrm{N}(\mathrm{Be}+\mathrm{B})]$ in NGC 663 with that of two rich clusters, NGC 7419 and NGC 2345. Since NGC 663, NGC 7419, and NGC 2345 are all rich, moderately young open clusters with ages between $31 \mathrm{Myr}$ and $79 \mathrm{Myr}$ (Kharchenko et al. 2013), NGC 663 could have a Be star fraction similar to that of NGC 7419 and NGC 2345. Interestingly, these authors showed that the $[\mathrm{N}$ $(\mathrm{Be}) / \mathrm{N}(\mathrm{Be}+\mathrm{B})]$ fraction of NGC 663 is $4.5 \%$, which is lower than that of NGC $7419(11 \%)$ and NGC $2345(26 \%)$. In spite of the fact that our result increases the $[\mathrm{N}(\mathrm{Be}) / \mathrm{N}(\mathrm{Be}+\mathrm{B})]$ fraction in NGC 663 from $4.5 \%$ to $6.8 \%$, the fraction is still the lowest among these three clusters.

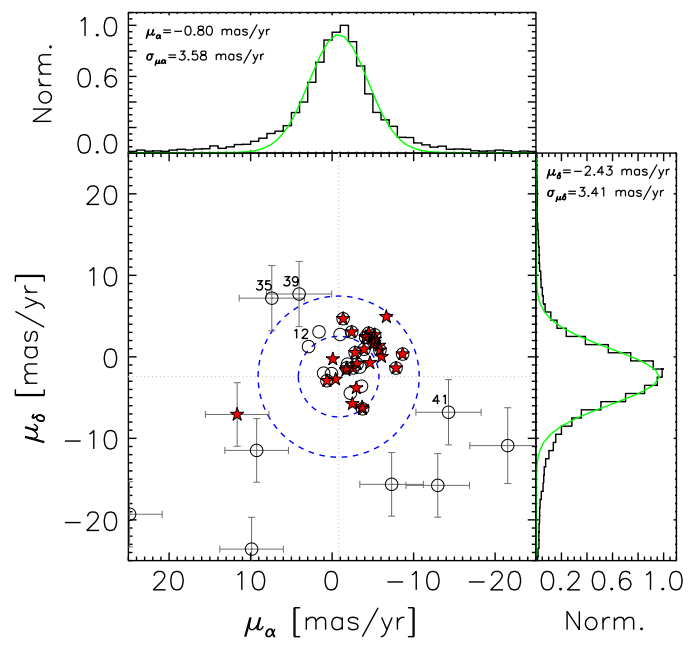

Figure 5. Stellar PMs of known Be stars and emission-line candidates toward NGC 663. The PM of NGC 663 is $\left(\mu_{\alpha}, \mu_{\delta}\right)=(-0.80,-2.43)$ mas year $^{-1}$. The inner and outer blue-dashed circles are $1 \sigma_{\mu}$ and $2 \sigma_{\mu}$ regions that define the probable PM membership. Symbols are the same as in Figure 2. The numbers represent the ID numbers of emission-line candidates listed in Table 1. The bars represent errors of PMs. The upper and right panels represent the $\sigma_{\mu \alpha}$ and $\sigma_{\mu \delta}$ distribution of stars within $40^{\prime} \times 40^{\prime}$; green lines show the Gaussian fitting to PMs.

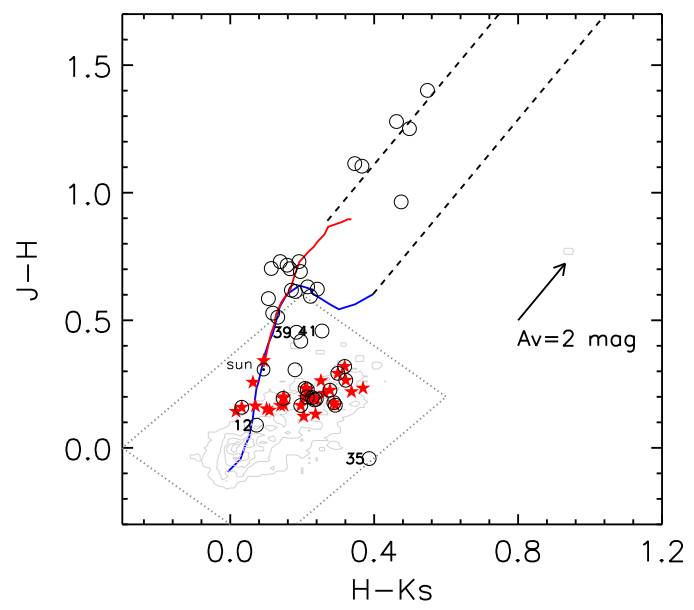

Figure 6. 2MASS color-color diagram in the cluster region. The red and blue curves show the giant and dwarf loci (Bessell \& Brett 1988) converted to the 2MASS system. The arrow represents the reddening direction (Rieke \& Lebofsky 1985) for typical Galactic interstellar extinction $\left(R_{V}=3.1\right)$, and the dashed lines encompass the region of reddened giants and dwarfs. The gray contours demonstrate known Be star distribution, and we define a dashed box to include all possible Be stars. Symbols are the same as in Figure 2.

It is notable that our procedure for membership identification is quite different from that of Mathew et al. (2008). Since they did not consider kinematic information when identifying memberships, we perform the same method (see Section 2.1.3) for NGC 7419 and NGC 2345 to identify membership and make a consistent comparison among these three clusters. Consequently, the ratio of Be stars to member stars $[\mathrm{N}(\mathrm{Be}) / \mathrm{N}$ (members)] of NGC 663, NGC 2345, and NGC 7419 are 3.5\% (34/959), 6.8\% (11/160), and 10.1\% (22/217). Once again, NGC 663 shows the lowest ratio among these three clusters. One possibility that would explain the low Be star fraction in NGC 663 is that the circumstellar material might be swept away by stellar winds from massive stars or supernova 
Table 2

Properties of Known Be Stars in NGC 663

\begin{tabular}{|c|c|c|c|c|c|c|c|c|c|c|c|}
\hline Name & $\begin{array}{l}\text { R.A. } \\
(\operatorname{deg})\end{array}$ & $\begin{array}{l}\text { Decl. } \\
\text { (deg) }\end{array}$ & $\begin{array}{c}\mu_{x} \\
\left(\text { mas year }^{-1}\right)\end{array}$ & $\begin{array}{c}\mu_{\delta} \\
\left(\text { mas year }^{-1}\right)\end{array}$ & $\begin{array}{c}\text { epm } \\
\left(\text { mas year }^{-1}\right)\end{array}$ & $\begin{array}{l}\mathrm{Ks} \\
(\mathrm{mag})\end{array}$ & $\begin{array}{l}J-H \\
(\mathrm{mag})\end{array}$ & $\begin{array}{c}H-K s \\
(\mathrm{mag})\end{array}$ & $\begin{array}{c}\text { EW }(\mathrm{H} \alpha) \\
(\AA)\end{array}$ & $\begin{array}{l}\text { Spectral } \\
\text { Type }\end{array}$ & Note \\
\hline MWC 428 & 26.325104 & 61.115692 & -5.05 & 1.57 & 2.00 & 8.602 & 0.196 & 0.209 & $\ldots$ & $\ldots$ & Saturated \\
\hline GG 102 & 26.648041 & 61.263290 & -4.64 & -0.74 & 2.02 & 8.880 & 0.132 & 0.236 & $\ldots$ & $\ldots$ & Saturated \\
\hline MWC 700 & 26.612782 & 61.167202 & -5.76 & 1.39 & 2.00 & 8.987 & 0.144 & 0.015 & $\ldots$ & $\ldots$ & Saturated \\
\hline VES 619 & 26.611837 & 61.128258 & -5.26 & 2.14 & 2.00 & 9.229 & 0.221 & 0.337 & $\ldots$ & $\ldots$ & Saturated \\
\hline BG 15 & 26.615303 & 61.207062 & -6.65 & 4.92 & 2.00 & 9.251 & 0.201 & 0.148 & $\ldots$ & $\ldots$ & Saturated \\
\hline MWC 698 & 26.497110 & 61.212685 & -3.69 & -6.16 & 2.89 & 9.425 & 0.148 & 0.109 & $\ldots$ & $\ldots$ & Saturated \\
\hline VES 616 & 26.525537 & 61.227573 & -2.48 & -5.76 & 2.00 & 9.645 & 0.234 & 0.368 & $\ldots$ & $\ldots$ & Saturated \\
\hline GG 110 & 27.096041 & 61.264717 & -2.96 & -3.82 & 2.00 & 9.704 & 0.168 & 0.149 & $\ldots$ & $\ldots$ & Saturated \\
\hline GG 104 & 26.750830 & 61.356567 & -2.56 & -1.37 & 2.00 & 9.899 & 0.200 & 0.213 & $\ldots$ & $\ldots$ & $\ldots$ \\
\hline GG 90 & 26.326494 & 60.969196 & -4.48 & 2.90 & 2.00 & 10.255 & 0.226 & 0.278 & $\ldots$ & $\ldots$ & $\ldots$ \\
\hline GC 97 & 26.483740 & 61.212574 & -1.35 & 4.68 & 1.50 & 10.262 & 0.266 & 0.321 & -27.6 & $\mathrm{~B} 2 \mathrm{~V}$ & $\ldots$ \\
\hline GG 95 & 26.443295 & 61.155804 & -1.79 & -1.36 & 2.00 & 10.321 & 0.198 & 0.228 & -38.8 & B1V & $\ldots$ \\
\hline GG 103 & 26.739870 & 61.027885 & -5.86 & 0.82 & 2.00 & 10.359 & 0.189 & 0.233 & $\ldots$ & $\ldots$ & $\ldots$ \\
\hline GG 98 & 26.584209 & 61.239319 & -6.07 & 0.02 & 2.00 & 10.402 & 0.213 & 0.262 & $\ldots$ & $\ldots$ & Blended \\
\hline GG 108 & 26.861519 & 61.145599 & -3.74 & -6.38 & 2.00 & 10.428 & 0.158 & 0.033 & -11.3 & B0-B1V & $\ldots$ \\
\hline GG 109 & 26.914015 & 61.305721 & -3.06 & -0.88 & 2.79 & 10.734 & 0.292 & 0.298 & -42.8 & B1V & $\ldots$ \\
\hline GG 101 & 26.648338 & 61.227543 & -4.11 & 2.39 & 2.00 & 10.762 & 0.318 & 0.318 & -54.0 & B1V & $\ldots$ \\
\hline VES 620 & 26.627619 & 61.241455 & -7.84 & -1.38 & 2.00 & 10.824 & 0.165 & 0.196 & -20.8 & B1V & $\ldots$ \\
\hline GG 99 & 26.612082 & 61.236439 & -5.19 & 1.75 & 2.00 & 10.880 & 0.153 & 0.101 & $\ldots$ & $\ldots$ & $\ldots$ \\
\hline GG 94 & 26.415096 & 61.216434 & -2.40 & 3.04 & 2.00 & 10.923 & 0.191 & 0.238 & -21.9 & $\mathrm{~B} 2 \mathrm{~V}$ & $\ldots$ \\
\hline D01-034 & 26.765589 & 61.292236 & -3.92 & 0.93 & 2.00 & 10.975 & 0.234 & 0.207 & -11.3 & $\ldots$ & $\ldots$ \\
\hline GG 93 & 26.407560 & 61.133095 & 0.63 & -2.93 & 2.00 & 11.102 & 0.167 & 0.291 & -34.9 & $\mathrm{~B} 2 \mathrm{~V}$ & $\ldots$ \\
\hline G32 & 26.619238 & 61.230675 & -4.15 & 2.47 & 2.00 & 11.338 & 0.125 & 0.203 & -7.9 & B3V & $\ldots$ \\
\hline BG 114 & 26.558376 & 61.228851 & -8.65 & 0.34 & 2.00 & 11.633 & 0.177 & 0.288 & -10.4 & B5V & $\ldots$ \\
\hline VES 624 & 26.748318 & 61.208199 & -5.21 & 2.70 & 2.59 & 12.032 & 0.227 & 0.215 & -26.8 & B5V & $\ldots$ \\
\hline L613 & 26.645269 & 61.107712 & -0.09 & -0.27 & 2.59 & 12.126 & 0.163 & 0.071 & -1.9 & B5V & $\ldots$ \\
\hline SAN 28 & 26.508623 & 61.250595 & -16.07 & -34.09 & 3.95 & 12.388 & 0.262 & 0.253 & -42.1 & B5V & CCD gap \\
\hline PKK 1 & 26.298687 & 61.173038 & 11.65 & -7.07 & 3.90 & 11.864 & 0.342 & 0.092 & $\ldots$ & $\ldots$ & $\ldots$ \\
\hline PKK 2 & 26.449562 & 61.273327 & -0.43 & -2.75 & 3.90 & 12.060 & 0.257 & 0.064 & $\ldots$ & $\ldots$ & $\ldots$ \\
\hline PKK 3 & 26.601690 & 61.177017 & -1.70 & -1.61 & 2.59 & 12.225 & 0.166 & 0.141 & -7.3 & B5-B7V & $\ldots$ \\
\hline PKK 4 & 26.823006 & 61.221569 & -2.82 & 0.51 & 3.90 & 12.961 & 0.194 & 0.148 & -6.5 & B5-B7V & $\ldots$ \\
\hline
\end{tabular}

Note. Column 1: name of known Be stars. Column 2: R.A. in degrees (J2000). Column 3: decl. in degrees (J2000). Column 4: proper motions of R.A. Column 5: proper motions of decl. Column 6: uncertainties of proper motions. Column 7: $K_{s}$-band magnitudes adopted from 2MASS. Column 8: $J-H$ magnitudes. Column 9: $H-K_{s}$ magnitudes. Column 10: H $\alpha$ equivalent widths adopted from Mathew \& Subramaniam (2011). If the objects have more than one observation at different epochs, we take the average equivalent widths. Column 11: spectral type adopted from Mathew \& Subramaniam (2011). Column 12: notes on objects.

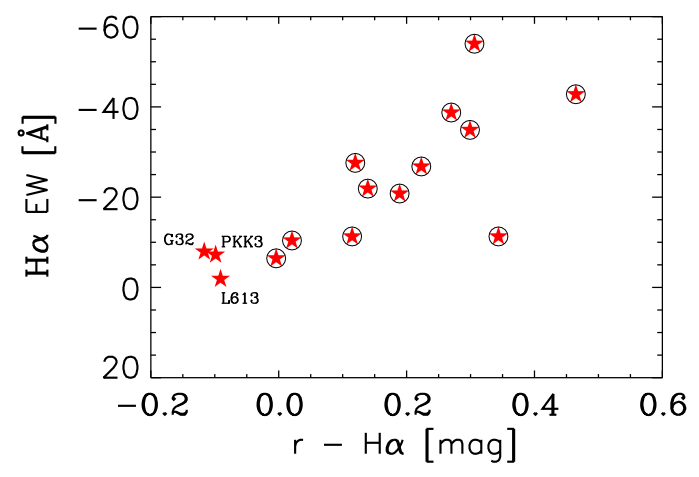

Figure 7. $\mathrm{EW}(\mathrm{H} \alpha)$ vs. $\mathrm{r}-\mathrm{H} \alpha$. Symbols are the same as in Figure 2. There is a correlation between the $\mathrm{EW}(\mathrm{H} \alpha)$ and $\mathrm{r}-\mathrm{H} \alpha$ values. Three known Be stars (PKK 3, L613, and G32) with weak H $\alpha$ strength (>-10 $\AA$ ) are not selected by our method.

explosions. Since NGC 663 could be a part of the stellar association Cas OB8, stellar winds from nearby massive stars or supernova events might remove the circumstellar material. Moreover, Pandey et al. (2005) reported that massive stars tend to lie in the center of NGC 663, and suggested that the mass segregation is primordial, e.g., due to the star formation process. When higher-mass stars are preferentially located toward the center of NGC 663, the circumstellar material might be swept up in the early stage of the star-forming process and thus cause the low Be star fraction.

\subsection{Spectral Type}

The spectral types of some Be stars in NGC 663 are present by Mathew \& Subramaniam (2011). It might be able to classify the stars based on the relative brightness of the stars with and without spectra. According to known spectra, stars with r-band instrumental magnitudes brighter than $8.5 \mathrm{mag}$ are classified as $\mathrm{B} 0-\mathrm{B} 2 \mathrm{~V}$, and the ones with magnitudes between 9 and 11 magnitudes are B5-B7 stars. Thus, we could classify the Be stars with magnitudes between 8.5 and 9 magnitudes as B3-B4 stars. Those candidates with $\mathrm{r}$-band magnitudes fainter than 11 mag might be late-type B or early-type A stars, e.g., B8-A0 stars. Consequently, we have $21 \mathrm{Be}$ stars in the B0-B2 type, including saturated stars, which should be brighter than 8.5 mag. We also have two Be stars in B3-B4 type, seven in B5-B7 type, and three in B8-A0 type. Our result shows a distribution of spectral types similar to that of previous studies; Mermilliod (1982) investigated the distribution of Be stars as a function of spectral types and found maxima at types of B1-B2 
and B7-B8 while Mathew et al. (2008) showed bimodal peaks in B1-B2 and B5-B7 for open clusters. Among the four newly discovered Be stars, one is classified as a B3-B4 star and three should be B8-A0 stars. We discover that three Be candidates belong to B8-A0 type, and the fraction of Be stars with a spectral type later than B5 is $21 \%$ (7/33) in NGC 663. Our results increase the fraction to $30 \%(10 / 33)$, which is consistent with that of other open clusters (Mathew et al. 2008).

\subsection{New Emission-line Candidates}

We have identified 42 emission-line stars within a $40^{\prime} \times 40^{\prime}$ field of NGC 663; 15 stars are known Be stars and 4 stars are newly discovered. Among the remaining 23 candidates, 2 candidates could be emission-line dwarf stars and 6 candidates could be giants in NGC 663. For the rest 15 candidates, we have also identified two stars as Be stars and 13 objects as emission-line dwarfs in the field that are not associated with the cluster. After cross-matching with the SIMBAD database, these 23 emission-line stars are presented for the first time.

\section{SUMMARY}

We apply $\mathrm{H} \alpha$ imaging photometry to identify 42 emissionline candidates in NGC 663. Four newly discovered Be stars have been identified as members using their CMDs and PMs. We also discover that one known Be star, SAN 28, should not be a cluster member due to its inconsistent PMs. Our results show that the number of Be stars in NGC 663 is 34, and the ratio of Be stars to member stars $[\mathrm{N}(\mathrm{Be}) / \mathrm{N}$ (members)] is $3.5 \%$. The low fraction of Be stars in NGC 663 might be a consequence of the effects of mass segregation and stellar winds or supernova explosions. Our results also reveal bimodal peaks in spectral types B0-B2 and B5-B7 for the Be population, which is consistent with the statistics in most star clusters. Finally, we discover 23 emission-line stars of nonmember Be stars, dwarfs, and giants. Our results suggest that the $\mathrm{H} \alpha$ photometry of the PTF data is very useful to search for emission-line stars. More Be stars in nearby clusters will be searched and investigated further to study the evolutionary scenario of Be phenomena.
We thank the referee for constructive comments. We also thank C. K. Chang, M. Kasiliwal, and H. C. Chen for useful discussions and information. This work is supported in part by the National Science Council of Taiwan under grants NSC 1012119-M-008-007-MY3 (W.-H.I.), NSC 102-2119-M-008-001 (W.-P.C.), and NSC101-2112-M-008-017-MY3 (C.-C.N.). This publication makes use of data products from the Two Micron All Sky Survey, which is a joint project of the University of Massachusetts and the Infrared Processing and Analysis Center/California Institute of Technology, funded by the National Aeronautics and Space Administration and the National Science Foundation.

\section{REFERENCES}

Bessell, M. S., \& Brett, J. M. 1988, PASP, 100, 1134

Chen, W. P., Chen, C. W., \& Shu, C. G. 2004, AJ, 128, 2306

Cutri, R. M., Skrutskie, M. F., van Dyk, S., et al. 2003, yCat, 2246, 0 Dambis, A. K., Mel'nik, A. M., \& Rastorguev, A. S. 2001, AstL, 27, 68 Fabregat, J., \& Torrejón, J. M. 2000, A\&A, 357, 451 Girardi, L., Bertelli, G., Bressan, A., et al. 2002, A\&A, 391, 195

Høg, E., Fabricius, C., Makarov, V. V., et al. 2000, A\&A, 355, L27

Keller, S. C., Grebel, E. K., Miller, G. J., \& Yoss, K. M. 2001, AJ, 122, 248

Kharchenko, N. V., Piskunov, A. E., Schilbach, E., Röser, S., \& Scholz, R.-D. 2012, A\&A, 543, 156

Kharchenko, N. V., Piskunov, A. E., Schilbach, E., Röser, S., \& Scholz, R.-D. 2013, A\&A, 558, A53

Law, N. M., Kulkarni, S. R., Dekany, R. G., et al. 2009, PASP, 121, L1395 Laher, et al. 2014, PASP, 126, 674

Lee, C.-D., \& Chen, W.-P. 2011, in IAU Symp., 272, Active OB Stars: Structure, Evolution, Mass Loss, and Critical Limits, ed. C. Neiner, G. Wade, G. Meynet, \& G. Peters (Cambridge: Cambridge Univ. Press), 366

Mathew, B., Subramaniam, A., \& Bhatt, B. C. 2008, MNRAS, 388, 1879

Mathew, B., \& Subramaniam, A. 2011, BASI, 39, 517

McSwain, M. V., \& Gies, D. R. 2005, ApJS, 161, 118

Mel'nik, A. M., \& Dambis, A. K. 2009, MNRAS, 400, 518

Mermilliod, J. C. 1982, A\&A, 109, 48

Pandey, A. K., Upadhyay, K., Ogura, K., et al. 2005, MNRAS, 358, 1290

Pigulski, A., Kopacki, G., \& Kołaczkowski, Z. 2001, A\&A, 376, 144

Rieke, G. H., \& Lebofsky, M. J. 1985, ApJ, 288, 618

Röser, S., Demleitner, M., \& Schilbach, E. 2010, AJ, 139, 2440

Sanduleak, N. 1979, AJ, 84, 1319

Sanduleak, N. 1990, AJ, 100, 1239

Townsend, R. H. D., Owocki, S. P., \& Howarth, I. D. 2004, MNRAS, 350, 189

Wisniewski, J. P., \& Bjorkman, K. S. 2006, ApJ, 652, 458

Zhang, P., Chen, P. S., \& Yang, H. T. 2005, NewA, 10, 325 